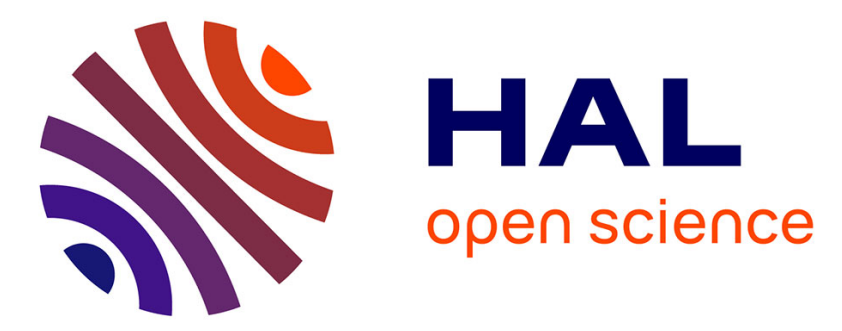

\title{
La place des dynamiques territoriales dans la régulation de l'orientation scolaire
}

Thierry Berthet, Stéphanie Dechezelles, Rodolphe Gouin, Véronique Simon

\section{To cite this version:}

Thierry Berthet, Stéphanie Dechezelles, Rodolphe Gouin, Véronique Simon. La place des dynamiques territoriales dans la régulation de l'orientation scolaire. Formation Emploi. Revue française de sciences sociales, 2010, L'orientation scolaire et professionnelle dans un monde incertain, 109, pp.37-52. 10.4000/formationemploi.2735 . hal-01796454

\section{HAL Id: hal-01796454 https://hal.science/hal-01796454}

Submitted on 20 May 2018

HAL is a multi-disciplinary open access archive for the deposit and dissemination of scientific research documents, whether they are published or not. The documents may come from teaching and research institutions in France or abroad, or from public or private research centers.
L'archive ouverte pluridisciplinaire HAL, est destinée au dépôt et à la diffusion de documents scientifiques de niveau recherche, publiés ou non, émanant des établissements d'enseignement et de recherche français ou étrangers, des laboratoires publics ou privés. 
Numéro 109 (janvier-mars 2010)

L'orientation scolaire et professionnelle dans un monde incertain

Thierry Berthet, Stéphanie Dechezelles, Rodolphe Gouin et Véronique Simon

\title{
La place des dynamiques territoriales dans la régulation de l'orientation scolaire
}

\begin{abstract}
Avertissement
Le contenu de ce site relève de la législation française sur la propriété intellectuelle et est la propriété exclusive de l'éditeur.

Les œuvres figurant sur ce site peuvent être consultées et reproduites sur un support papier ou numérique sous réserve qu'elles soient strictement réservées à un usage soit personnel, soit scientifique ou pédagogique excluant toute exploitation commerciale. La reproduction devra obligatoirement mentionner l'éditeur, le nom de la revue, l'auteur et la référence du document.

Toute autre reproduction est interdite sauf accord préalable de l'éditeur, en dehors des cas prévus par la législation en vigueur en France.
\end{abstract}

\section{revues.org}

Revues.org est un portail de revues en sciences humaines et sociales développé par le CLEO, Centre pour l'édition électronique ouverte (CNRS, EHESS, UP, UAPV).

\section{Référence électronique}

Thierry Berthet, Stéphanie Dechezelles, Rodolphe Gouin et Véronique Simon, «La place des dynamiques

territoriales dans la régulation de l'orientation scolaire », Formation emploi [En ligne], 109 | janvier-mars 2010, mis en ligne le 01 mars 2012. URL : http://formationemploi.revues.org/index2735.html

DOI : en cours d'attribution

Éditeur : La documentation française

http://formationemploi.revues.org

http://www.revues.org

Document accessible en ligne à l'adresse suivante : http://formationemploi.revues.org/index2735.html Ce document est le fac-similé de l'édition papier.

Cet article a été téléchargé sur le portail Cairn (http://www.cairn.info).

\section{CAIR N}

Chercher. Repérer. Avancer.

Distribution électronique Cairn pour La documentation française et pour Revues.org (Centre pour l'édition électronique ouverte)

(c) Tous droits réservés 


\section{DOSSIER}

\section{La place des dynamiques territoriales dans la régulation de l'orientation scolaire}

Par Thierry Berthet, Stéphanie Dechezelles, Rodolphe Gouin, Véronique Simon*

\section{L'orientation scolaire est liée aux dynamiques territoriales et à la régulation des services d'orientation. L'autonomie locale des opérateurs délimite leurs modes de coordination et les processus d'orientation.}

Le dispositif français d'orientation s'ordonne autour d'un clivage marquant entre orientation scolaire et post-scolaire. D'un côté, les structures d'orientation dédiées au public scolaire relèvent, pour l'essentiel, du réseau des Centres d'information et d'orientation (CIO) du ministère de l'Éducation nationale ; de l'autre, une série de réseaux offrent aux jeunes sortis du système scolaire et aux adultes, salariés ou demandeurs d'emploi, des prestations en orientation. Ces deux ensembles se distinguent, non seulement par le statut de leur public mais aussi, et c'est plus rarement évoqué, par leur rapport au territoire. Les réseaux d'orientation post-scolaire, notamment ceux participant au service public de l'emploi (agences locales pour l'emploi, missions locales, centres AFPA - Association nationale pour la formation professionnelle des adultes) s'inscrivent résolument dans les dynamiques locales de la relation formationemploi. Tel n'est pas nécessairement le cas des acteurs de l'orientation scolaire, conformément aux représentations courantes qui en font un dispositif national relevant de la politique d'éducation conduite par son
* Thierry Berthet est chargé de recherche CNRS à l'université de Bordeaux, au SPIRIT (Science Politique Relations Internationales Territoires, UMR 5116). Ses recherches portent sur les politiques publiques d'orientation, la territorialisation des politiques d'emploi et la régionalisation de la formation professionnelle. II a récemment publié : Maiiten Bel et al. (2009), "Proximité et relation emploiformation : au carrefour des disciplines », Espaces et sociétés 1-2, pp. 136-137 et pp. 33-46; Berthet T. (2008), " Les enjeux de l'évaluation territoriale des politiques publiques ", Informations sociales, $n^{\circ}$ 150, octobrenovembre.

Stéphanie Dechezelles est maître de conférences en science politique à l'Institut d'études politiques d'Aixen-Provence, rattachée au CHERPA (Croyance, Histoire, Espace, Régulation Politique et Administrative) et associée au SPIRIT (UMR 5116 ) - université de Bordeaux. Elle travaille sur l'engagement politique des jeunes et les partis de "droite", principalement en Italie. Elle a notamment publié : avec T. Berthet, R. Gouin et V. Simon, "Orientation: la parole aux élèves", NEF-Céreq, $n^{\circ} 34,(2008)$; «Les acteurs locaux de l'orientation: 
un exemple aquitain », NEF-Céreq n³5 (2008) et "Le système d'orientation. Entre choix individuels et contraintes d'action publique », NEF-Céreq n³6 (2008).

Rodolphe Gouin est docteur en science politique, chercheur associé au SPIRIT. Ses recherches portent sur l'épistémologie des sciences sociales, les rapports des sciences cognitives à la science politique et les approches cognitives des politiques publiques et de l'action collective. II a récemment publié : "De l'usage des sciences cognitives dans l'analyse des politiques publiques », (avec Jean-Baptiste Harguindéguy) Swiss Review for Political Science, 13(3), (2007); "Temporaliser l'analyse des mobilisations 》, avec Gael Franquemagne et Bénédicte Récappé, Thierry Berthet, Olivier Costa, Rodolphe Gouin et al. (dirs.) in Les nouveaux espaces de la régulation politique, L'Harmattan (2007).

Véronique Simon est chargée d'études au centre régional associé Céreq / SPIRIT de Bordeaux. Ses travaux portent sur les politiques d'orientation et l'offre de formation continue en France. Elle a publié récemment, avec T. Berthet, S. Dechezelles et R. Gouin : "Orientation : la parole aux élèves », NEF-Céreq, $n^{\circ} 34$, septembre 2008 et " Les acteurs locaux de l'orientation : un exemple aquitain », NEF-Céreq, n³5, septembre 2008.

ministère de tutelle ${ }^{1}$. Son ancrage territorial scolaire s'efface ainsi derrière une vision stato-centrée. Cette perspective, qui accorde à l'intervention de l'État un rôle dominant, est largement partagée par la littérature scientifique et technique ainsi que par les opérateurs de terrain.

Prenant le contre-pied de cette vision commune, l'objectif de cet article est de montrer comment les dynamiques territoriales constituent un facteur clé pour comprendre l'orientation scolaire, et notamment ses modes de régulation.

Cet angle d'approche sur l'orientation scolaire est décalé au regard d'une littérature scientifique qui s'est largement focalisée sur trois dimensions. Une première série de travaux interroge sa fonction sociale. Ce rôle est à l'origine d'une série de recherches sociologiques interrogeant sa place dans les processus de ségrégation sociale et scolaire, et plus largement l'articula-

${ }^{1}$ Cette vision s'avère aujourd'hui fermement établie ; rappelons qu'elle a soutenu, par exemple, le rejet, par les personnels de l'Éducation nationale, de la décentralisation du réseau des CIO aux conseils régionaux en 2003. tion entre les transformations de l'appareil scolaire et du système d'orientation (Girard, 1953 ; Grignon, 1968, Berthelot, 1978 et 1993 ; Duru-Bellat, 1988 ; Broccolichi, 1995 ; Buisson-Fenet, 2005 ; Boudesseul \& Grelet, 2008). Une deuxième série de travaux questionne la nature particulière de la relation de conseil en orientation. Elle a été largement explorée par de nombreux travaux, en premier lieu ceux conduits à l'INETOP (Institut national d'étude du travail et de l'orientation) et plus généralement dans les cercles des professionnels de l'orientation (Masson, 1997 ; Guichard et Huteau, 2005 ; Danvers, Wulf et Aubret, 2006). Enfin, l'histoire et l'organisation du système d'orientation scolaire ont fait l'objet de plusieurs recherches et études. Sur le versant historique, les travaux s'attachent à retracer les étapes de la construction de ce système, notamment dans ses liens denses et complexes avec le système éducatif (Caroff 1987 ; Prost, 1997 ; Sénécat, 2004). Par ailleurs, de nombreux rapports et études relatifs à l'organisation du système d'orientation français corroborent les constats de « dysfonctionnements » récurrents de celui-ci mais sans en explorer les causes profondes, notamment dans une perspective territorialisée (Hénoque \& Legrand, 2004 ; IGEN-IGAENR, 2005 ; Reiss, 2007 ; Haut Comité Éducation, 2008).

Il ressort de cette littérature sur l'orientation scolaire que la place des dynamiques territoriales demeure sous-étudiée. Pourtant, l'intérêt d'une approche de la relation orientation/territoire est double. D'une part, elle rétablit le lien distendu entre une histoire de l'orientation marquée par son ancrage local et les réflexions contemporaines sur son organisation et sa réforme. D’autre part, elle permet de dégager l'analyse d'une tension opposant les perspectives marquées soit par une posture « déterministe », liée à la logique de la reproduction sociale, soit par une approche «stratégiste » de l'orientation, conçue comme fondée sur l'autonomie et les stratégies des individus. En effet, il nous semble que le dispositif et les processus d'orientation scolaire sont adossés à des pratiques situées qui doivent se comprendre dans leur contexte local (Champollion, 2008). Pour approfondir cette réflexion, nous tenterons de montrer, dans cet article, que l'ancrage local de l'orientation scolaire prend un relief particulier au prisme de trois aspects liés : l'autonomie organisationnelle des services, la multiplicité des liens 
de partenariats tissés par les établissements avec leur environnement local et le rôle du territoire dans les processus concrets d'orientation des élèves.

Dans une première partie, nous montrerons que l'histoire de l'orientation scolaire s'enracine dans les territoires, en dépit d'un récit l'inscrivant dans une épopée nationale qui articule démocratisation/ massification de l'éducation et planification de la relation formation-emploi. Dans une deuxième partie, nous étudierons, sur la base d'une enquête de terrain réalisée en Dordogne, la nature et le rôle des coordinations localisées des acteurs de l'orientation (cf. encadré 1). Enfin, nous soulignerons, dans une troisième partie, que ces deux éléments - importance de la structuration territoriale de l'orientation scolaire et rôle des coordinations d'acteurs - contribuent à lier les processus d'orientation au territoire.

\section{Encadré 1 \\ Méthodologie}

Le matériau empirique mobilisé est issu d'une étude sur les politiques et pratiques d'orientation scolaire, à la demande du conseil régional d'Aquitaine et avec la collaboration des services du rectorat de Bordeaux. Elle a été conduite par les politistes du centre associé du Céreq en Aquitaine. Le rapport général d'enquête est disponible en ligne sur le site http:// www.cereq.fr/pdf/Net-doc4 1 .pdf

Menée en 2007-2008 dans trois zones d'emploi d'un département rural, la Dordogne, cette recherche articule méthodes quantitative et qualitative. En premier lieu, 57 entretiens semi-directifs ont été conduits aux niveaux local, départemental et régional, auprès de décideurs publics et d'opérateurs en matière d'orientation scolaire. Au niveau local, proviseurs, professeurs principaux, animateurs de Centre de documentation et d'information et conseillers d'orientation psychologues ont été interrogés dans chaque établissement. En complément, des entretiens visant à saisir la relation des établissements scolaires avec leur environnement ont été menés avec les responsables de différentes structures (Agence locale pour l'emploi, mission locale, bureau information jeunesse, espaces Économie-Emploi, centre d'information et d'orientation). Au niveau départemental et régional, les entretiens ont été menés auprès des services académiques (inspection d'académie et rectorat), de la Direction de l'Éducation du conseil régional, de la Direction régionale du Travail, de l'Emploi et de la Formation professionnelle, de la coordination régionale des missions locales(*), et enfin, au national, ces rencontres se sont adressées à des inspecteurs généraux de l'Éducation nationale en charge de l'orientation et des responsables syndicaux notamment. Ces entretiens fournissent un matériau anonymisé relatif aux modes de régulation du système.

Par ailleurs, ces entretiens sont complétés par des données issues d'un questionnaire /constitué de 60 questions, ouvertes ou fermées, à choix multiples ou non). L'échantillon retenu pour cette enquête était composé de 351 élèves (163 filles et 188 garçons) de six classes (3e Générale, 3e Agricole, 2nde Générale, BEP - brevet d'études professionnelles - 1 re année, BEP 2e année (terminale), BEPA - brevet d'études professionnelles agricole - (terminale) de l'Éducation nationale et hors Éducation nationale (deux collèges, un lycée d'enseignement général et technologique, un lycée polyvalent, un lycée professionnel, un lycée d'enseignement général technologique agricole, une maison familiale rurale, un centre de formation des apprentis). Les données déclaratives recueillies par questionnaire portent ainsi principalement sur les représentations des élèves quant à leur parcours scolaire. Elles ont été traitées par une société spécialisée et exploitées avec le concours du centre associé du Céreq en Basse Normandie.

(*) Les missions locales sont des structures locales intervenant notamment sur l'insertion sociale et professionnelle des jeunes de moins de 25 ans et libérés de l’obligation scolaire. Elles assument ainsi, pour cette catégorie de publics, des fonctions d'accueil, d'information et d'orientation, et sont de ce fait notamment partie prenante du service public de l'emploi. 


\section{ORIENTATION ET TERRITOIRE : UN LIEN HISTORIQUE ÉVOLUTIF MAIS PERMANENT}

L'histoire de l'orientation professionnelle et scolaire, en France, s'enracine dans celle des grands traumatismes politiques et mutations économiques des XIXe et $\mathrm{XX}^{\mathrm{e}}$ siècles et, bien évidemment, dans l'histoire du système éducatif. Les phases de reconstruction succédant aux deux guerres mondiales ont constitué, de ce point de vue, des moments clés de redéfinition des missions de l'enseignement et de l'orientation. Une première phase voit se développer les structures locales d'orientation professionnelle qui, dans un second temps, seront progressivement arrimées à l'appareil scolaire, tout en demeurant faiblement régulées par l'administration centrale.

\section{Les premières structurations de l'orientation à partir d'initiatives locales}

Le développement de l'orientation professionnelle se discute, dès 1920, dans les débats parlementaires consacrés au rattachement de l'enseignement technique au ministère de l'Instruction publique. Ces discussions aboutissent à la promulgation du décret du 26 septembre 1922 instituant l'orientation professionnelle, en France, sous la forme d'offices (privés ou publics) autonomes venant appuyer le travail des offices publics de placement ${ }^{2}$. Si les offices d'orientation s'inscrivent dans un dispositif national - adossé à la création d'un système spécifique d'inspection (1924) et intégrant deux initiatives privées : l'Institut national d'orientation professionnelle (1927) et le Bureau universitaire de statistique (1932) - ils constituent avant tout des structures locales. Émergeant d'une grande diversité d'initiatives et de configurations locales, animés par des personnels aux statuts divers et pouvant être fonctionnaires comme agents des collectivités territoriales (communes, départe-

\footnotetext{
2 De ce moment fondateur, qui voit l'orientation naître « en dehors de l'école " (IGEN-IGAENR, 2005, p. 3), émerge une règle qui fera date : les services d'orientation n'effectuent pas directement le placement des jeunes ; ils aident les offices de placement qui assument cette responsabilité. Cette logique de conseil aux organismes qui décident de l'affectation des jeunes, en emploi comme en scolarité, demeure une constante du système français d'orientation.
}

ments), ces offices d'orientation sont rattachés soit à des offices de placement soit à des organismes relevant des milieux économiques locaux (chambre consulaire, association, syndicat). Entre 1920 et 1928, 110 centres voient le jour (Caroff, 1987, p. 91).

Une réforme engagée à la veille de la Seconde Guerre mondiale vient encadrer, sans le disqualifier, ce pluralisme qui accorde une place importante aux acteurs locaux. Le décret-loi du 24 mai 1938 ainsi que ses textes d'application prévoient en effet principalement la mise en place d'un secrétariat départemental d'orientation professionnelle et la transformation des « offices » en « centres » d'orientation professionnelle. Les secrétaires d'orientation professionnelle, ancêtres des actuels IEN-IO (Inspecteurs de l'Éducation nationale en charge de l'information et de l'orientation), sont des agents nommés par le ministre chargé de l'Enseignement technique, placés sous l'autorité du préfet et rémunérés par l'État. Le secrétariat qu'ils dirigent est quant à lui financé par les départements. À l'orée de la Seconde Guerre mondiale, le dispositif français d'orientation repose ainsi organisationnellement sur deux piliers : les secrétariats départementaux et les centres d'orientation professionnelle. Ce dispositif, visant le développement d'une orientation professionnelle, est «sous-tendu par un humanisme visant à l'émancipation individuelle et à l'harmonie sociale " qui laisse aux acteurs locaux une large capacité d'initiative et d'organisation. «Durant cette période, l'État est peu interventionniste... [et] se contente donc de créer un cadre législatif et réglementaire puis de contrôler la mise en œuvre " (Sénécat, 2004, pp. 10 et 11).

La logique de reconstruction qui suit la Seconde Guerre mondiale accompagne un volontarisme fort de l'État dans le champ de l'orientation professionnelle, qui prend la forme d'une nationalisation des structures d'orientation. Cette dynamique supporte l'émergence progressive de l'orientation scolaire. Elle s'incarne d'abord, d'un point de vue financier, par un investissement important de l'État dans les structures départementales. Cet effort financier est relayé par une refonte du dispositif national institué par l'article 11 de la loi de finances de 1952, elle-même complétée par les décrets du 10 octobre 1955 et du 6 avril 1956. Au terme de cette réforme, les centres d'orientation professionnelle relèvent d'une forme hybride entre 
une structure locale, qui reste à la charge financière des départements et des communes, et un service déconcentré du ministère. En effet, le décret de 1956 assure l'entrée de leurs personnels dans la fonction publique et la création de trois catégories d'agents répartis en deux corps : inspecteurs d'un côté, directeurs de centres et conseillers d'orientation, de l'autre. Cette distinction perdure aujourd'hui. Le directeur de centre d'orientation est ainsi, dès le départ, institué comme « un conseiller d'orientation qui assure de surcroît des responsabilités de direction » (Caroff, 1987, p. 139). À côté de ces centres d'orientation scolaire et professionnelle publics subsistent encore des centres privés relevant le plus souvent d'organismes consulaires. Si les centres publics vont progressivement s'imposer comme modèle, ils conserveront néanmoins un ancrage local dans les départements et pour certains dans les communes. La réforme Berthoin (1959) va marquer le développement de l'orientation scolaire en structurant les procédures qui associeront désormais les centres départementaux d'orientation scolaire et professionnelle et leurs conseillers, mais n'aura guère d'effets sur la structuration du système d'orientation au niveau local. Néanmoins, les changements introduits au niveau de l'administration centrale de l'Éducation nationale ${ }^{3}$ marquent les prémices d'un détachement de l'orientation scolaire du champ professionnel. Elle sera par la suite fermement arrimée à l'appareil éducatif initial.

\section{Le rattachement aux établissements scolaire aboutit à une régulation de moins en moins centralisée}

Les réformes conduites entre 1970 et 1973 structurent fortement, tout en l'autonomisant, le système d'orientation et ses procédures. Cette réorganisation s'entend tant d'un point de vue fonctionnel que territorial. Au niveau de l'académie, une nouvelle figure émerge : le chef des services académiques d'information et d'orientation (CSAIO) qui, sous l'autorité du recteur, aura pour tâche d'animer la réflexion régionale et de coordonner la politique académique d'orientation auprès des services départementaux. Au

\footnotetext{
${ }^{3}$ Les instances nationales pilotant le système d'orientation se structurent ainsi en se détachant de la Direction générale de l'Enseignement technique pour relever désormais de la Direction générale de l'Organisation et des Programmes scolaires, ce qui marque plus fortement l'ancrage scolaire de l'orientation.
}

niveau départemental, les IEN-IO ont pour fonction de relayer les actions décidées au niveau académique. Au niveau local, cette réforme consacre la transformation des centres publics d'orientation scolaire et professionnelle en centres d'information et d'orientation (CIO). Ce changement de dénomination n'affecte pas, sur le fond, les missions et l'organisation des CIO. En revanche, c'est dans la relation au territoire que le changement est le plus perceptible. En effet, cette réforme introduit la détermination d'une zone infra-départementale qui deviendra le ressort géographique du CIO : le district scolaire ${ }^{4}$. Le rattachement des CIO aux districts affirme l'arrimage des centres d'orientation aux dynamiques des établissements scolaires et éloigne les structures d'orientation des logiques départementales et/ou municipales ayant prévalu jusqu'alors. Cette dynamique est renforcée par la possibilité ouverte alors de transformer les CIO départementaux en CIO d’État placés sous l'autorité du recteur 5 . Depuis, la structure du dispositif d'orientation scolaire n'a guère évolué et demeure fortement intégrée à l'appareil.

Pourtant, il ne faudrait pas en conclure que cette restructuration, qui consacre de manière formelle l'avènement de l'orientation scolaire comme politique nationale stato-centrée, s'est traduite par une régulation fortement centralisée et une coupure des territoires. En effet, la régulation de ce dispositif se caractérise par une faible densité du pilotage national et une grande autonomie des acteurs locaux. Ainsi, nos observations sur les processus d'orientation en Aquitaine ont souligné la grande autonomie locale dont bénéficient les opérateurs de l'orientation scolaire (Berthet et alii, 2008). En premier lieu, les CSAIO ne sont pas les supérieurs hiérarchiques des IEN-IO. Comme le note ce CSAIO : " Au nom du Recteur, il y $a$ une fonction d'animation auprès des CIO, sachant que la chaîne hiérarchique opérationnelle, c'est l'Inspecteur d'Académie qui formellement dans les textes est le 'patron' entre guillemets des conseillers

\footnotetext{
${ }^{4}$ Créés en 1965, les districts scolaires correspondent à l'aire de recrutement des élèves d'un ou plusieurs lycées et servent de cadre géographique à l'affectation des élèves. Cette affectation des CIO au niveau territorial des districts, par l'article 5 du décret 71-541 du 7 juillet 1971, sera réaffirmée par la circulaire n $80-099$ relative à l'organisation des centres d'information et d'orientation.

5 Cette disposition entraîne la coexistence, jusqu'à aujourd'hui, de ces deux types de $\mathrm{CIO}$, et les inégalités de dotation financière qui en découlent.
} 
et des CIO. Donc la fonction d'animation du CSAIO est très fonctionnelle, au moins de ce point de vue là. » Par ailleurs, les IEN-IO n'exercent pas de tutelle hiérarchique sur les CIO. Qui plus est, depuis la fin des années 70, ils n’inspectent plus les conseillers d'orientation psychologues (CO-P) : « Officiellement, aucune [autorité hiérarchique]. C'est-à-dire que je n'ai par rapport aux personnels d'orientation, qu'un lien fonctionnel (...). Il n'y a pas d'inspection, non. (...) Je ne note jamais un conseiller, c'est uniquement le directeur qui va les noter, et administrativement, pas sur sa prestation (...). Il n'y a pas d'évaluation de la prestation d'un conseiller d'orientation. » (entretien avec un IEN-IO). Enfin, l'orientation scolaire ne fait plus l'objet d'un pilotage national soutenu de la part du ministère de l’Éducation nationale. La Division de l'information et de l'orientation, autrefois rattachée à la Direction de l'Enseignement scolaire, a été supprimée en 1989. "On a supprimé la Division de l'orientation comme on a supprimé le service de la formation continue et à partir de ce moment là, il n'y a plus eu d'animation (...) À partir de ce moment là, l'orientation a été administrée, elle n'a plus été animée » (entretien avec un IGEN, dernier chef de la Division de l'orientation). En 2005, il ne subsistait au ministère qu'une Mission à l'orientation, composée de quatre personnes ${ }^{6}$, pour piloter le réseau des $578 \mathrm{CIO}$ et annexes. Un des principaux enseignements du regard rétrospectif sur l'orientation scolaire consiste ainsi à relever un paradoxe qui tient au fait que l'intégration de l'orientation scolaire au système éducatif s'est rapidement accompagnée d'une perte de régulation centralisée sur ses opérateurs de terrain. Ainsi, après que les CIO ont été intégrés dans l'appareil scolaire, l'administration centrale ne s'est pas dotée d'une organisation permettant de réguler le fonctionnement de ce réseau maillant le territoire. Cela s'est traduit par une forte autonomie des CIO de manière générale et des conseillers d'orientation psychologues en particulier. La faible densité de la régulation hiérarchique verticale, et l'autonomie relative des CIO qui découle de cet héritage historique contribuent à expliquer l'importance des mécanismes de coordination horizontale et les configurations localisées dans lesquelles ils s'inscrivent.

\footnotetext{
${ }^{6}$ Rattachée au directeur de l'Enseignement scolaire (IGENIGAENR, 2005, p. 13).
}

\section{DES COORDINATIONS MULTIPLES ET TERRITORIALISÉES}

La structuration organisationnelle et la faiblesse de la régulation hiérarchique placent les personnels des CIO dans une position de relative autonomie, où la question de la coordination de leur intervention avec celle des autres acteurs de l'orientation scolaire comme post-scolaire s'avère centrale. Cette partie est consacrée à l'analyse des modes de collaboration établis entre opérateurs de l'orientation. Elle déborde ainsi le cadre strict de l'orientation scolaire pour embrasser plus largement les partenariats qui se nouent, au sein et hors des établissements, autour de la question de l'orientation des élèves.

Deux types de travaux théoriques sont mobilisés ici pour analyser des coordinations d'acteurs. D'un côté, les travaux de science politique consacrés aux politiques territoriales ont démontré l'importance croissante des mécanismes de coordination d'acteurs et la manière dont ils tendent à se substituer aux modes de régulation sectoriels et stato-centrés ayant prévalu jusqu'au début des années 80 (Duran, 1999 ; Mériaux, 2005 ; Faure et Négrier, 2007 ; Smith, 2009). Ces travaux sur l'action publique locale corroborent l'hypothèse selon laquelle la coordination des personnels des CIO avec celle des autres acteurs de l'orientation scolaire comme post-scolaire devient centrale en raison de la faiblesse de la régulation hiérarchique qui caractérise ce secteur ${ }^{7}$. Les sociologues de l'action publique et les analystes des politiques publiques (Faure, Leresche, Narath et Muller, 2007 ; Lascoumes et le Galès, 2005) signalent également l'importance des représentations et des instruments dans les mécanismes de gouvernance multiniveaux qui se structurent ainsi. De leur côté, les économistes de la proximité soulignent l'importance d'un certain nombre de variables dans l'analyse des coordinations d'acteurs, en insistant notamment sur les rôles de la proximité géographique et des conditions de coopération créées par les formes organisationnelles et

\footnotetext{
7 Ne subsistent en effet que ces coordinations qui, dès lors, deviennent simultanément fonctionnellement centrales (au lieu d'être construites dans une périphérie dont le centre serait l'administration centrale) et géographiquement locales (parce que liées fortement à la nature de leur environnement qui diffère d'un territoire à l'autre).
} 
institutionnelles (Pecqueur et Zimmermann, 2004 ; Talbot, 2008 ; Bel et Berthet, 2009). Nous prêterons donc, dans cette partie, une attention particulière aux formes organisationnelles, au rôle des représentations et des instruments dans les coordinations des acteurs de l'orientation, en distinguant selon que les coordinations étudiées sont internes ou externes aux établissements.

\section{Des coordinations internes aux établissements}

Dans le champ scolaire, et plus précisément au sein des établissements, les conseillers d'orientation psychologues (CO-P) ne sont pas les seuls intervenants en matière d'orientation scolaire ${ }^{8}$. Ils collaborent avec les chefs d'établissements qu'ils conseillent en matière d'orientation et avec les enseignants, dont le rôle en matière d'orientation n'a cessé de croître à mesure que la régulation centrale du réseau des CIO s'affaiblissait. Les effets d'image et de positionnement jouent fortement à ce niveau. D’un côté, les conseillers d'orientation psychologues sont identifiés par les parents comme des acteurs incontournables de l'orientation, même si cette image est parfois brouillée : «La demande des parents identifie très clairement le CO-P comme la personne en charge de l'orientation. On a l'impression que les élèves mettent leur orientation en stand by quand qu'il n'y a pas de COP. Ils ont encore cette image de l'orientation faite par un conseiller." (entretien avec un proviseur). Mais leur implication dans la vie de l'établissement apparaît variable en fonction de facteurs individuels ou de la stratégie du responsable de CIO, comme en témoigne ce proviseur : "C'est fonction de l'implication des CO-P, moi j'ai connu de tout, ils ont vraiment un effet structurant quand ils sont dynamiques, quand ils sont impliqués (...). Nous sommes demandeurs, on sait le boulot que peut faire un CO-P, ça manque. J'ai $v u$ des CO-P très investis, faisant vraiment partie de l'équipe pédagogique. Ça ne se négocie pas (avec le CIO), vous avez tant d'heures, c'est telle personne, point. (...) Le directeur du CIO s'investit énormément et quand je veux quelque chose pour un élève.

\footnotetext{
$864 \%$ des élèves interrogés dans le cadre de notre recherche estiment avoir été aidés dans leurs choix par leurs parents, ce qui est de loin le score le plus élevé ; viennent ensuite les professeurs principaux, à $35 \%$, les CO-P à $28 \%$, les amis à $28 \%$, les journées portes ouvertes à $20 \%$, et les stages en entreprise à $18 \%$.
}

je passe par lui. » Ce niveau d'implication est aussi lié aux effectifs de conseillers et d'élèves ainsi qu'à la marge d'autonomie dont disposent les premiers dans l'organisation de leur travail.

Du côté des enseignants, l'action en matière d'orientation est structurée par la proximité avec les familles : «En tant que professeur principal, je suis la parole de l'équipe et cette équipe, à un moment donné, pose un diagnostic final et un conseil sur l'orientation, enfin un avis. (...). Moi depuis que je suis professeur principal de 3e, c'est ma 6e année, je ne me souviens pas d'une famille qui ait dit "bon j'arrête, je vais voir le chef d'établissement", parce que de toute façon ici, la politique du chef d'établissement, c'est de faire confiance à ses professeurs principaux » (entretien avec un professeur principal de collège). L'orientation n'est toutefois pas sans poser de problèmes aux enseignants, comme en témoigne ce professeur principal de lycée : «L'orientation, qu'on ne nous fasse pas porter des casquettes en plus, le boulot de prof, c'est déjà quelque chose, être conseiller d'orientation, c'est autre chose. Qu'on nous donne des outils oui, mais qu'on ne nous fasse pas porter le poids. On est très mal placés... on a toujours fait partie des bons élèves! ».

Quant aux chefs d'établissements, leur tâche est complexe et articule, dans le pilotage stratégique de leur établissement, les contraintes liées à l'affectation des élèves, le partenariat avec les acteurs locaux ${ }^{9}$ et l'animation de l'équipe pédagogique : "On est des sortes de "super surveillants" pour les élèves, on est des hybrides. Et on ne connaît pas du tout leurs résultats. On parlait d'indicateurs donc, je vous cache pas qu'on essaie de travailler afin de faire évoluer les indicateurs dans le sens qui semble raisonnable... je pense au taux de redoublement, je pense effectivement au taux d'accès aux classes de première, je pense au taux d'abandon en cours de seconde, je pense à tous

\footnotetext{
${ }^{9}$ En matière d'affectation, les chefs d'établissement doivent intégrer les contraintes liées aux places disponibles dans les établissements de formation (à commencer par le leur) pour assumer leur responsabilité de décideur final en matière d'orientation des élèves. Ils doivent aussi cultiver les liens avec les acteurs locaux (politiques, économiques, associatifs). Ainsi, dans le domaine spécifique de l'orientation, la mise en œuvre de l'option « découverte professionnelle » (3 heures) en classe de troisième, a supposé que les principaux de collège œuvrent, de concert avec les enseignants responsables de l'option, à la densification des relations avec les acteurs économiques locaux.
} 
ces indicateurs là, donc effectivement l'idée c'est de faire passer peut-être un peu plus d'élèves de collège en seconde et d'élèves de seconde vers le baccalauréat. (...) Ce qu'on essaie de mettre en place, c'est impulser cette politique là, inciter les enseignants à faire passer les élèves parfois un peu fragiles mais en accompagnant ces élèves là. » (entretien avec un proviseur).

Dans la coordination de ces interventions internes aux établissements émergent des enjeux organisationnels complexes, où se croisent des professionnalités présentées comme complémentaires : "Le discours qu'on va leur tenir, ça va être un discours de prof tandis que eux [les CO-P NdA] ils voient la souffrance du gamin, nous on ne peut pas en tenir compte. Ils peuvent dire au conseiller d'orientation "moi je ne fiche rien", à nous (les profs principaux) ils ne peuvent pas le dire. » (entretien avec un professeur principal de lycée). L'établissement est spontanément le niveau où se construisent et se régulent ces coordinations qui supposent une articulation étroite entre direction d'établissement et CIO. Les principaux outils sur lesquels s'appuient ces collaborations sont les projets des établissements et leurs interconnexions ${ }^{10}$.

\section{Des coordinations externes multiniveaux}

L'animation locale de l'orientation et des transitions scolaires suppose aussi la coordination des établissements sur les territoires. Les proximités spatiale et organisationnelle déterminent ces collaborations qui s'organisent sur plusieurs échelles territoriales en fonction des types de coordination visés.

Dénomination locale du bassin d'éducation et de formation, au niveau infra départemental, la « zone d'animation pédagogique » (ZAP) est présentée par les acteurs locaux interrogés comme un premier niveau où s'organisent les partenariats pédagogiques. Désignant une équipe territoriale chargée de coordonner et d'organiser une série de travaux impulsés par ses membres ou l'Inspecteur d'académie en fonc-

\footnotetext{
${ }^{10}$ Il faut noter qu'à l'échelle des districts, une politique d'orientation coordonnée pourrait exister dans la mesure où, en principe, CIO et établissements sont liés par un " projet d'orientation », mais la nature de l'intervention (face-à-face individuel privilégié) et le grand nombre d'établissements à charge des conseillers ne permettraient pas le temps de concertation nécessaire.
}

tion des orientations définies par le Recteur, la ZAP constitue une structure souple d'animation pédagogique, comme l'illustre ce propos : «Au niveau de la ZAP, on a mis en place une formation à l'attention des professeurs principaux de seconde dans le cadre de la liaison troisième/seconde. Les professeurs de seconde vont rencontrer, dans l'établissement, au mois de janvier, ceux de troisième afin d'échanger sur les compétences exigibles d'un élève de seconde et d'avoir une image un peu plus juste de l'élève pour les deux parties. Et il y a aussi des échanges par discipline : français, mathématiques et histoire-géo. » (Responsable d'établissement, lycée)

Elle constitue, de ce fait, un niveau privilégié d'interaction entre établissements scolaires. Les relations à l'intérieur de la ZAP sont jugées satisfaisantes par nos interlocuteurs : " Au niveau de la ZAP, cela fonctionne assez bien entre nous » (entretien avec un principal). Niveau d'articulation pédagogique, la ZAP est aussi un niveau où se développent, grâce au travail d'animation de l'IEN-IO, un certain nombre d'actions et d'échanges relatifs à l'orientation : " Au niveau de la ZAP, il y a des réunions lycée/collèges et des réunions spécifiques pour l'orientation " (entretien avec un proviseur). Pour structurer l'action de prévention et de remédiation envers les publics les plus en difficulté, la coordination pédagogique des établissements est un enjeu clé où « il est indispensable de réintroduire une logique territoriale, à savoir que la mission générale d'insertion (MGI) et toute la problématique des ruptures scolaires doivent se faire au sein de la ZAP » (entretien avec un CSAIO). La structure ad hoc des interventions respecterait ainsi, selon cet interlocuteur en charge de l'animation régionale de l'orientation, un ordre ascendant des degrés territoriaux par type de coordination : "l'établissement, la ZAP, le département et l'échelon académique, seulement après en coordination et en animation générale ».

Mais il manque un barreau à cette échelle : les structures d'orientation post-scolaire. Les relations ne sont pourtant pas absentes ; elles sont même régulièrement évoquées par nos interlocuteurs. "Avec la mission locale, on a fait une tentative de rapprochement à travers le Carrefour des métiers (...). Le CIJ (Centre d'Information Jeunesse), ils sont à l'écoute de nos besoins ; via le comité d'éducation à la santé et à la citoyenneté (CESC), si on a besoin qu'une action soit 
mise en place, ils la mettent en place » (Responsable d'établissement, collège). Les équipes des différents établissements signalent toutes, à des degrés et des intensités variables, des formes de partenariat avec d'autres structures d'orientation ${ }^{11}$, des organismes de la relation formation-emploi ${ }^{12}$ et des entreprises locales qui constituent autant de ressources pour l'orientation : "l'Espace Économie Emploi de Lalinde a travaillé avec les scolaires, pour une sensibilisation aux métiers du bâtiment » (Responsable Espace Économie Emploi). Pour autant, ces partenariats demeurent généralement ponctuels et peu formalisés s'agissant des établissements de l’Éducation nationale. Pour les organismes de formation, ces relations sont plus routinières : « Maison de l'emploi, Espace Économie Emploi, Mission locale, (...) aujourd'hui sur le bergeracois on se connaît tous et ça marche bien. (...) Le partenariat, c'est plus une histoire d'hommes parce qu'au niveau institutionnel, c'est compliqué : il y a deux chambres consulaires, trois avec l'agriculture, il y a des associations et nous on est municipal. Mais au niveau du territoire, on travaille bien » (Responsable d'établissement, CFA). Évoqués comme un facteur de développement d'une orientation fondée sur une meilleure interconnaissance des voies de formation et d'insertion professionnelle, ces partenariats avec l'environnement territorialisé des établissements scolaires demeurent encore souvent entravés par des systèmes de représentations bien ancrés : "Nous on n'est pas du monde de l'entreprise, ça ne nous correspond pas $d u$ tout, on n'est pas attiré ni intéressé par ça. Çà nous demande un effort d'aller vers cet univers là. De même, ils ont tellement d'a priori sur l'Éducation nationale que ce sont deux univers qui ne se rencontrent pas forcément " (entretien avec un professeur principal, lycée). «On ne travaille pas pareil, on est sur l'insertion professionnelle » (entretien avec un responsable de mission locale). Pour autant, ces coor-

\footnotetext{
11 Agences Locales pour l'Emploi (Pôle Emploi), Missions locales, Espaces Economie Emploi. Créés à partir de 1992, les Espaces Economie Emploi sont une spécificité de la Dordogne. Ils constituent des structures de connaissance des territoires et de partenariat des acteurs issus du SPE (service public de l'emploi), des entreprises et des structures de formation.

12 Notamment les Centres de formation des apprentis et les Maisons familiales rurales (MFR). Les MFR sont des structures de l'économie sociale contractualisées par le ministère de l'Agriculture et/ou conventionnées par le Conseil régional ou l’État, qui accueillent des jeunes dès la classe de $4^{e}$ ou de $3^{e}$ de l'enseignement agricole, ainsi que des adultes en formation par alternance.
}

dinations avec les acteurs de l'orientation et de l'insertion professionnelle se développent. «On travaille en étroite collaboration avec les établissements scolaires de Bergerac, collèges, lycées, lycées professionnels, lycées agricoles, CFA (...) Là, on a travaillé en partenariat avec le CIO bien sûr, la Mission locale. Notamment, on avait fait ça pour tout ce qui est métiers de la fonction publique » (responsable d'un Bureau d'Information Jeunesse). Ces coordinations sont marquées du sceau du local parce que fortement dépendantes des liens construits par les établissements avec leur environnement territorial immédiat. Elles s'inscrivent, de ce fait, dans des dynamiques locales singulières. La zone d'emploi ( $c f$. tableau 1) est le niveau auquel se réfèrent les acteurs locaux quand ils décrivent ces partenariats, alors qu'elle forme initialement un cadre purement administratif et descriptif : «si on prend les lycées professionnels du Bergeracois, on est sur des offres de formation qui sont liées à notre bassin d'emploi » (Professeur principal, collège).

Des observations que nous avons conduites en Dordogne, il ressort ainsi que les coordinations établies localement s'organisent spontanément sur un mode multiniveaux. Les opérateurs de l'orientation investissent en effet des échelles territoriales différentes selon le type de coordination qu'ils développent. C'est ainsi bien la finalité de la coordination développée qui justifie qu'elle se structure sur des scènes locales différentes, allant de l'établissement à la zone d'emploi, et selon des logiques où prédominent des dynamiques locales singulières. Les prémisses des économistes de la proximité et des analystes de l'action publique se vérifient bien ici en ce que la proximité géographique s'articule aux dispositifs organisationnels territorialisés existants et selon des processus immédiatement multiniveaux. Si on ajoute à ces coordinations locales l'articulation avec un niveau régional qui devient sans cesse plus prégnant, on peut résumer ces coordinations multiniveaux des acteurs de l'orientation dans le tableau $2^{13}$.

Le dispositif d'orientation scolaire s'avère ainsi historiquement construit dans un rapport étroit avec les

\footnotetext{
13 Précisons que cette proposition de mise en œuvre ne vise pas à remettre en cause les modalités d'organisation des institutions existantes (par exemple, en délégitimant le niveau départemental des inspections de l'Éducation nationale ou l'action des conseils généraux en matière collégiale).
} 
dynamiques territoriales, et ce positionnement singulier soutient des coordinations multiples et territorialisées qui investissent une pluralité de niveaux. Mais il importe surtout de relever qu'en matière d'orientation scolaire, comme dans le champ de l'orientation post- scolaire (CCPR, 2004 ; Jousserandot, 2004 ; Berthet \& Gayraud, 2003), l'autonomie des opérateurs, les dynamiques territoriales et les mécanismes de coordination des acteurs locaux s'articulent pour influencer les processus d'orientation des individus.

Tableau 1

Définition des principaux zonages évoqués

\begin{tabular}{|l|l|}
\hline \multicolumn{1}{|c|}{ Zonage } & \multicolumn{1}{c|}{ Définition } \\
\hline District scolaire & $\begin{array}{l}\text { Aire de recrutement d'un ou plusieurs lycées et niveau d'exercice des ClO (centre d'information } \\
\text { et d'orientation) (Circulaire du } 18 \text { juin 1965). }\end{array}$ \\
\hline $\begin{array}{l}\text { Bassin d'éducation et } \\
\text { de formation (BEF) }\end{array}$ & $\begin{array}{l}\text { Les missions du bassin s'organisent autour de trois pôles : pilotage et animation pédagogique, } \\
\text { gestion déconcentrée des ressources humaines, renforcement des partenariats de proximité } \\
\text { (Circulaire du } 20 \text { juin 200 1). } \\
\text { I est dénommé zone d'animation pédagogique (ZAP) dans l'académie de Bordeaux }\end{array}$ \\
\hline $\begin{array}{l}\text { Zone d'emploi } \\
\text { et travaillent. Effectué conjointement par l'Insee et les services statistiques du ministère du Travail, } \\
\text { le découpage en zones d'emploi constitue une partition du territoire adaptée aux études locales } \\
\text { sur l'emploi et son environnement. Les déplacements domicile-travail constituent la variable de } \\
\text { base pour la détermination de ce zonage. }\end{array}$ \\
\hline
\end{tabular}

Source : auteurs.

\section{Tableau 2}

\section{Niveaux territoriaux et types de coordination des actions d'orientation}

\begin{tabular}{|c|c|c|c|}
\hline $\begin{array}{l}\text { Niveau } \\
\text { territorial }\end{array}$ & Type de coordination pertinente & Acteurs & Orientation \\
\hline Établissement & $\begin{array}{l}\text { Coordination pédagogique au } \\
\text { sein de l'établissement. }\end{array}$ & $\begin{array}{l}\text { Chef d'établissement, conseiller } \\
\text { principal d'éducation, enseignants } \\
\text { (professeurs principaux notamment), } \\
\text { CO-P (conseillers d'orientation } \\
\text { psychologues). }\end{array}$ & $\begin{array}{l}\text { Orientation scolaire interne } \\
\text { à l'établissement et vers } \\
\text { les filières qui y sont } \\
\text { développées. }\end{array}$ \\
\hline $\begin{array}{l}\text { Bassin d'édu- } \\
\text { cation et de } \\
\text { formation } \\
\text { (BEF) }\end{array}$ & $\begin{array}{l}\text { Coordination pédagogique } \\
\text { entre établissements d'enseigne- } \\
\text { ment Éducation nationale et hors } \\
\text { Éducation nationale. }\end{array}$ & $\begin{array}{l}\text { IEN-IO (Inspecteurs de l'Éducation } \\
\text { nationale en charge de l'informa- } \\
\text { tion et de l'orientation), animateurs } \\
\text { du BEF, chefs d'établissements, } \\
\text { directeurs de CIO (centre d'informa- } \\
\text { tion et d'orientation). }\end{array}$ & $\begin{array}{l}\text { Orientation scolaire interne } \\
\text { et en direction des autres } \\
\text { établissements d'enseigne- } \\
\text { ment du bassin, intervention } \\
\text { sur le décrochage scolaire. }\end{array}$ \\
\hline Zone d'emploi & $\begin{array}{l}\text { Coordination stratégique avec } \\
\text { environnement économique et } \\
\text { liée à l'orientation postscolaire. }\end{array}$ & $\begin{array}{l}\text { Missions locales, Agences locales } \\
\text { pour l'emploi, entreprises; cham- } \\
\text { bres consulaires, etc. }\end{array}$ & $\begin{array}{l}\text { Orientation scolaire/ } \\
\text { postscolaire. }\end{array}$ \\
\hline Région & $\begin{array}{l}\text { Coordination politique entre les } \\
\text { réseaux d'orientation et entre } \\
\text { politiques d'orientation et autres } \\
\text { politiques publiques (dévelop- } \\
\text { pement économique, insertion, } \\
\text { éducation, formation profession- } \\
\text { nelle, emploi). }\end{array}$ & $\begin{array}{l}\text { Rectorat, Conseil régional, } \\
\text { Coordination régionale des } \\
\text { missions locales, Préfecture, } \\
\text { Chambres consulaires régionales, } \\
\text { DRAAF (Direction régionale de l'Ali- } \\
\text { mentation, de l'Agriculture et des } \\
\text { Forêts), DRJS (Direction régionale } \\
\text { de la Jeunesse et des Sports), etc. }\end{array}$ & $\begin{array}{l}\text { Orientation tout au long } \\
\text { de la vie. }\end{array}$ \\
\hline
\end{tabular}

Source : auteurs. 


\section{OFFRE LOCALE DE FORMATION ET MOBILITÉ : DES ENJEUX STRATÉGIQUES POUR L'ORIENTATION}

Les liens entre dynamiques territoriales et coordination des acteurs de l'orientation scolaire d'un côté, processus d'orientation des élèves, de l'autre, sont complexes et multiples. Les présenter de manière exhaustive nécessiterait de larges développements. Néanmoins, des enquêtes de terrain conduites en Aquitaine émergent deux questions liées qui s’avèrent déterminantes pour comprendre ces contraintes jouant sur les processus d'orientation : celle du cadre constitué par l'offre locale de formation d'une part, et celle de la mobilité géographique des élèves, d’autre part. Rappelons que les variations locales dont il est question ici ne sont pas un vain mot et qu'elles produisent des effets en termes d'orientation des élèves. À titre d'exemple, le département étudié, la Dordogne, possède des taux de passage en première générale inférieurs et des taux de réorientations supérieurs aux moyennes régionales et nationales ${ }^{14}$. Au niveau infra-départemental, l'examen des taux de passage $2^{\text {nde }} / 1^{\text {re }} \mathrm{GT}$ souligne une variation importante selon les établissements, qui va de 75 à 92 \%.

\section{L'offre locale de formation comme horizon}

Dans la grande majorité, lors de nos entretiens avec les acteurs de l'orientation scolaire, les choix d'orientation des élèves s'effectuent souvent en fonction de l'offre de formation de proximité. La géographie des choix recouperait ainsi celle de l'offre environnante, comme le confirme cet IEN-IO : "si on connaît la carte du secteur d'un lycée, a priori on sait quelle orientation vont prendre les lycéens de ce lycée ». Ce mécanisme d'orientation de proximité relève autant du comportement des élèves que du comportement de ceux qui les aident à s'orienter : « les élèves ne font pas véritablement de choix personnels. Ils font ces choix

14 Taux de passage en $1{ }^{\text {re }}$ générale après une 2 de générale et technologique : Dordogne $53 \%$, Aquitaine $60 \%$, France $57 \%$; Taux de réorientation en fin de $2^{\text {de }}$ générale et technologique : Dordogne $8 \%$, Aquitaine $5 \%$, France $6 \%$. Source : Inspection académique de Dordogne. parce que l'établissement est à coté, c'est fonction de la proposition locale. Après c'est aussi peut-être par méconnaissance des filières qui existent. Pourquoi ? Parce que les enseignants ne savent pas non plus » (proviseur lycée professionnel du Bergeracois). Pour les enseignants, la référence en matière d'orientation est ainsi souvent l'offre de formation la plus immédiate et proche.

Concernant les élèves, et en dépit d'une faible mobilité, ce discours dominant sur un déterminisme de la proximité mérite d’être relativisé. En effet, les réponses aux questionnaires des jeunes périgourdins enquêtés invitent à un point de vue plus nuancé. « Travailler sans changer de région » est ainsi la dernière des attentes que les élèves formulent à propos du métier qu'ils souhaitent exercer ${ }^{15}$. Cette représentation n'est pourtant pas liée à la question de la poursuite d'études et à l'obligation pour les élèves d'être mobiles en raison d'une faible offre locale de formation. En effet, les élèves qui ne souhaitent pas poursuivre leurs études ne sont que 20 \% à déclarer s'arrêter après l'obtention du diplôme préparé parce que la poursuite d'études les obligerait à déménager. L'hypothèse d'une préférence des jeunes pour la proximité et les différentes formes de déterminisme liées à l'offre locale de formation qui sont évoquées par les opérateurs de l'orientation doivent ainsi être nuancées, au moins du point de vue des représentations des élèves. Mais ces représentations se heurtent à une série de principes de réalité, particulièrement ceux liées à la gestion de l'appareil éducatif.

Les contraintes liées à l'affectation - c'est-à-dire à la gestion des flux d'élèves en fonction des places disponibles dans les établissements - ont toute leur importance, même si « la contrainte d'affectation est à ne pas confondre avec une erreur d'orientation » (Directeur de CIO). La carte locale de l'offre de formation initiale influence en effet fortement les décisions d'orientation scolaire via ses modes de gestion technique et entraîne un brouillage de la distinction entre les pratiques d'orientation et d'affectation. Construite dans une perspective de gestion administrative des

\footnotetext{
15 À la question "Quand vous pensez au métier que vous voulez faire, vous voulez qu'il vous permette de : ", les élèves enquêtés disposaient de la possibilité de cocher trois réponses parmi les dix réponses possibles, dont l'item «travailler sans changer de région ». Cette dernière réponse obtient le plus faible score et n’a été choisie que par 1,5 \% des élèves.
} 
flux d'élèves, l'affectation confère aux procédures d'orientation une dimension fortement prescriptive, en particulier pour les jeunes les plus en difficulté. Pour ces derniers, les décisions d'orientation viennent parfois valider un choix contredisant leurs vœux, y compris lorsque ceux-ci, parfois, sont réalistes au regard de leur " performance scolaire ». Si l'orientation est un champ des possibles, l'affectation quant à elle "se heurte à une réalité finie, constituée par la carte des formations en évolution très faible (entre 5 et $10 \%$ de modifications annuelles), dans laquelle elle doit répartir les élèves en fonction de choix qui sont souvent déséquilibrés. C'est surtout le cas des enseignements professionnels » (Andréani et Lartigue, 2006, p. 165). Ce rôle décisif des procédures d'affectation dans la décision finale d'orientation peut donc s'analyser comme un effet de pilotage de l'orientation par l'offre de formation.

Mais, au-delà des contraintes qu'elle génère sur les choix des élèves, la référence au territoire et à la carte des formations est aussi un outil stratégique pour les différents acteurs locaux du système éducatif. Ainsi, les établissements sont susceptibles de mobiliser la dimension territoire (en accroissant par exemple les acceptations de dérogations d'élèves issus de départements proches) pour maintenir le volume de leur offre de formation (classes et sections). Du côté de l'Inspection d'académie, la carte scolaire peut constituer un moyen de pression (par refus de ces mêmes dérogations par exemple) envers les établissements jugés trop sélectifs ${ }^{16}$.

Enfin, les caractéristiques socio-éducatives du territoire sont mobilisées par les opérateurs locaux de l'orientation scolaire (comme les IEN-IO ou les chefs d'établissements par exemple) pour légitimer le maintien de la carte scolaire, alors même que des effectifs d'élèves à la baisse pourraient amener à sa remise en cause. «En termes de carte scolaire, on va avoir une position qui a intérêt à mettre en avant des particularismes du département pour avoir quelque chose. Parce que sinon, comme la population est relativement stable, on n'obtiendra jamais rien. Donc on est un peu vécu à l'académie comme des défenseurs de la

16 «Certains établissements se démarquent par des taux de passage (entre classes) systématiquement en dessous du niveau de la demande » et sont " plus sélectifs que d'autres » (entretien avec un IEN-IO). carte scolaire du département et à ce moment là, on joue bien département contre académie ou une mise en tension des deux » (entretien avec un IEN-IO). En d'autres termes, la référence au territoire et à la carte scolaire peut se voir instrumentée pour soutenir les objectifs des différents agents. Ainsi, dans la régulation de l'orientation, la place qu'occupe la gestion de la carte des formations n'est pas seulement administrative et technique mais prend parfois les traits d'un enjeu stratégique local. Elle soulève aussi, pour les départements ruraux notamment, la question clé de la mobilité des élèves.

\section{La mobilité comme facteur de ségrégation}

En milieu rural, la mobilité géographique du public conditionne l'accès aux formations supérieures au niveau $\mathrm{V}^{17}$ et dessine un jeu de contraintes par l'offre. Plus que choisir une filière, l'orientation peut signifier " partir ou rester ", puisque notamment le choix des élèves est limité par la faiblesse de l'offre des bac en lycée rural. De la même façon, pour les jeunes à la recherche d'un premier emploi, l'absence de mobilité peut constituer un handicap si le bassin d'emploi de leur lieu de résidence s'avère faiblement dynamique et pauvre en opportunités d'emploi. La mobilité spatiale devient un pré-requis pour accéder au niveau $\mathrm{IV}^{18}$ quand l'offre de formation est peu développée au-delà du niveau V. Elle structure aussi pour partie la difficulté d'accès à l'emploi des publics les moins qualifiés (Arrighi, 2004).

En l'absence de mobilité des jeunes, le territoire, quand il est démuni de ressources en possibilités d'emploi et de formation, se dresse comme une contrainte qui génère des choix d'orientation contraints par la nécessité de s’inscrire dans les opportunités localement disponibles en termes de filières de formation ou d'insertion professionnelle ${ }^{19}$. En formation initiale

\footnotetext{
17 Niveau de formation équivalent à celui du brevet d'études professionnelles (BEP) et du certificat d'aptitude professionnelle (CAP).

18 Niveau de formation équivalent à celui du baccalauréat, du brevet de technicien (BT), du brevet supérieur d'enseignement commercial (BSEC) .

19 Même si le taux d'appel des décisions reste faible, le terme " contraint » peut renvoyer au processus en amont de la formulation de la demande. Comme une forme d'autocensure lors de la formulation de ses vœux, l'élève anticipe le choix dont il pense qu'il lui serait de toute façon imposé par la suite. La contrainte
} 
par exemple, nos observations en Dordogne montrent que si le choix de tel type de bac impose l'internat, les élèves de seconde ne disposant pas des moyens suffisants s'orienteront plus facilement vers une première présente au sein de leur établissement.

À l'instar de la question de la proximité, les représentations des élèves à propos de la poursuite d'études incitent à nuancer les points de vue et à s'interroger sur le rôle des contraintes agissant sur leurs choix. Ainsi, les élèves de la voie professionnelle interrogés lors de notre enquête révèlent des préférences pour les études allant au moins jusqu'au bac professionnel, voire au-delà (75 \% des élèves de BEP/CAP dans les établissements de l'Éducation nationale n'envisagent pas d'arrêter leurs études après l'obtention de leur diplôme). Pour les moins mobiles, orientation scolaire et post-scolaire basculent aisément du registre du conseil vers celui, nettement plus directif, de la prescription. Les interventions des acteurs de l'orientation, et notamment des enseignants, se centrent alors sur la modification des comportements individuels, en amenant les jeunes à opter pour une formation «plus réaliste » ou un métier vers lequel ils ne seraient pas spontanément allés, en bref à changer d'idée.

Mais à travers le contexte spatial de leurs choix se profile aussi le poids de l'origine sociale : «Les apprentis ne sont pas d'un milieu social très aisé. Ils n'ont pas tous la mobylette au départ, ils l'ont à la fin, ils se la paient » (entretien avec un directeur d'un CFA - centre de formation d'apprentis). Pour les acteurs interrogés, les choix d'orientation sont situés géographiquement et la mobilité est aussi une question économique : "Les enfants en difficulté, ils n'iront pas à Périgueux faire un BEP industriel, ce n'est pas la peine, les familles ne pourront pas payer. (...) S'ils habitent à Terrasson [situé à $55 \mathrm{~km}$ de Périgueux], ils ont le train presque gratuit, mais s'ils habitent sur les hauteurs, à quelques kilomètres, ils n'iront pas, parce que personne ne pourra les conduire à la gare » (entretien avec un proviseur de lycée).

La contrainte socio-spatiale pèse ici lourdement : niveaux de qualification contraints par l'offre, offre de formation supérieure extrêmement réduite entraînant une moindre qualification des moins mobiles. Au regard des exigences du marché du travail, un risque accru de vulnérabilité pèse sur les moins qualifiés. Dans ce contexte, mobilité et promotion révèlent bien leur étymologie commune ${ }^{20}$ et, au bout du compte, le terme immobile a bien pour synonyme interdit : «S'ils ne sont pas mobiles, il ne faut pas qu'ils tentent un niveau $I V$ », (entretien avec un directeur d'un CFA). Le local n'est pas sans risque.

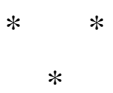

L'objectif de cet article est de rappeler la densité des liens qui unissent les services d'orientation scolaire (CSAIO/IEN-IO/CIO), les dynamiques territoriales et la régulation des pratiques d'orientation des élèves. Il vise aussi à en proposer une analyse qui mette en relation les effets de l'autonomie locale de ses opérateurs sur leurs modes de coordination et les processus d'orientation. Il a ainsi été possible de montrer, à partir d'un retour sur l'histoire des structures d'orientation scolaire en France, l'importance des dynamiques locales qui ont accompagné leur constitution, et de souligner que les modalités de leur intégration dans l'appareil éducatif, au cours du dernier tiers du $\mathrm{XX}^{\mathrm{e}}$ siècle, ont laissé perdurer une large autonomie locale de leurs agents. Cette indépendance relative rend d'autant plus prégnante la question des modes de coordination établis localement par les acteurs de l'orientation scolaire et post-scolaire (CO-P conseillers d'orientation psychologues -, enseignants, responsables d'établissements, centres de formation d'apprentis, maisons familiales rurales, missions locales, etc.). À ce sujet, on peut observer que ces collaborations se structurent sur des échelles territoriales distinctes selon la nature des coordinations mises en place. Des formes spontanées de gouvernance se développent ainsi sur plusieurs niveaux territoriaux, au gré des nécessités et des opportunités de partenariat que saisissent ou créent les acteurs locaux. En termes d'analyse des politiques publiques, ce constat remet clairement en question la recherche d'un niveau unique de régulation des pratiques d'orientation.

Cela dit, s'intéresser aux formes organisationnelles ne suffit pas. Il faut de surcroît souligner que ces 
formes, les stratégies des acteurs de l'orientation et les ressources disponibles qui sont spécifiques à chaque territoire, contraignent les processus d'orientation des élèves et agissent, en milieu rural notamment, sur les opportunités de choix et de promotion qui s'offrent à eux.

Nous avons esquissé, sur la base d'une recherche locale, ces différentes pistes qui apparaissent, à bien des égards, fécondes tant d'un point de vue théorique qu'analytique. Il nous semble ainsi qu'un programme de recherche se dessine autour d'une réelle prise en compte du rôle des facteurs liés aux modes de régulation et aux dynamiques des territoires. Une telle démarche présente notamment l'intérêt de décloisonner l'étude de l'orientation scolaire d'une approche centrée exclusivement sur l'institution scolaire ou les modes d'élaboration des choix individuels. En d'autres termes, l'étude des processus d'orientation dans les établissements ne devrait pas faire l'économie d'une analyse « en contexte ».

\section{Bibliographie}

Andréani F. et Lartigues P. (2006), L'orientation des élèves. Comment concilier son caractère individuel et sa dimension sociale, Paris, Armand Colin.

Arrighi J.-J. (2004), « Les jeunes dans l'espace rural : une entrée précoce sur le marché du travail ou une migration probable », Formation Emploi, n ${ }^{\circ} 87$.

Bel M., Berthet T. (2009), " Proximité et relation emploi-formation: au carrefour des disciplines ", Espaces et Sociétés, nº 136-137, pp. 33-46.

Berthelot J.-M. (1978), « Exode agricole et scolarisation : Analyse différentielle des modalités de scolarisation d'une population scolaire du second degré dans un département rural sur-scolarisé », Revue française de sociologie, Vol. $19, \mathrm{n}^{\circ} 1$ (janvier-mars), pp. 103-124.

Berthelot J.-M. (1993), École, orientation, société, Paris, PUF.

Berthet T., Dechezelles S., Gouin R. et Simon V. (2008), Les acteurs locaux de l'orientation: un exemple aquitain, Céreq, $N E F \mathrm{n}^{\circ} 35$.

Berthet T., Gayraud L. (2003), «Gouverner l'action publique aux marges, l'exemple de l'orientation professionnelle » in M. Bel, O. Mériaux et P. Méhaut,
La décentralisation de la formation professionnelle en France. Quels changements dans la conduite de l'action publique ?, Paris, L'Harmattan.

Boudesseul G., Grelet Y. (2008), « Choix d'orientation et logiques institutionnelles ", Céreq, NEF, $n^{\circ} 32$.

Broccolichi S. (1995), « Orientations et ségrégations nouvelles dans l'enseignement secondaire », Sociétés contemporaines, 21, pp. 15-27.

Buisson-Fenet H. (2005), « Des professions et leurs doutes: procédures d'orientation et décisions de "réorientation" scolaire en fin de seconde », Sociétés

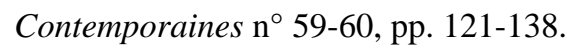

Caroff A. (1987), L'organisation de l'orientation des jeunes en France, Issy les Moulineaux, Éditions EAP.

Champollion P. (2008), « La territorialisation du processus d'orientation en milieux ruraux isolés et montagnards : des impacts du territoire à l'effet de territoire ", Éducation \& Formations, $\mathrm{n}^{\circ} 77$ novembre, pp. 43-54.

CCPR (2004), Pour une politique régionale coordonnée d'accueil, d'information et d'orientation, 
Rapport remis par R. Regnault et D. Vernedoub au Comité de Coordination des Programmes régionaux d'Apprentissage et de Formation Professionnelle Continue.

Danvers F., Wulf C., Aubret J. (2006), Modèles, concepts et pratiques en orientation des adultes, Villeneuve d'Ascq, Presses Universitaires du Septentrion.

Duran P. (1999), Penser l'action publique, Paris, LGDJ.

Duru-Bellat M. (1988), Le fonctionnement de l'orientation : genèse des inégalités sociales à l'école, Paris, Delachaux-Niestlé.

Faure A., Leresche J.-P., Muller P. et Narath S. (2007), Action publique et changements d'échelle: Les nouvelles focales du politique, Paris, L'Harmattan.

Faure A. et Négrier E. (éd.) (2007), Les politiques publiques à l'épreuve de l'action locale. Critiques de la territorialisation, Paris, L'Harmattan.

Girard A. (1953), « L’orientation et la sélection des enfants d'âge scolaire dans le département de la Seine », INED-Population, 8e année, $\mathrm{n}^{\circ} 4$ (octobre décembre), pp. 649-672.

Grignon C. (1968), « L’orientation scolaire des élèves d'une école rurale ", Revue française de sociologie, vol. 9, pp. 218-226.

Guichard J. et Huteau M. (2005), L'orientation scolaire et professionnelle, Paris, Dunod.

Haut Conseil de l'Éducation (2008), L'orientation scolaire, Bilan des résultats de l'école.

Hénoque M., Legrand A. (2004), L'évaluation de l'orientation à la fin du collège et au lycée. Rêves et réalités de l'orientation, Rapport du Haut Conseil de l’Évaluation de l'École.

IGEN-IGAENR (2005), Le fonctionnement des services d'orientation, Rapport $n^{\circ} 2005-101$ remis au ministre de l'Éducation nationale, de l'Enseignement supérieur et de la Recherche.

Jousserandot F. (2004), L'action publique d'aide à l'orientation 1981-2001, Thèse de doctorat en science politique, IEP de Grenoble.

Lascoumes P., Le Galès P. (2005), Gouverner par les instruments, Paris, Presses de Sciences Po.

Masson P. (1997), «Élèves, parents d'élèves et agents scolaires dans le processus d'orientation ", Revue française de sociologie, vol. 38, $n^{\circ} 1$, pp. 119-142.

Mériaux O. (2005), « Le débordement territorial des politiques sectorielles », in L'action publique et la question territoriale, édité par Faure A. et Douillet A.-C., Grenoble, Presses Universitaires de Grenoble.

Prost A. (1997), Éducation, société et politiques. Une histoire de l'enseignement de 1945 à nos jours, Éditions Seuil.

Pecqueur B., Zimmermann J.-B. (2004), Économie de proximité, Lavoisier, Paris, Hermès Science Publications.

Reiss F. (2007), Avis présenté au nom de la commission des affaires culturelles, familiales et sociales sur le projet de loi de finances pour 2008, Paris, Assemblée nationale, 11 octobre.

Sénécat J. (2004), «Historique de l'orientation professionnelle en France », Conférence présentée dans le cadre des Rencontres de la DESCO, http:// eduscol.education.fr/D0122/orientation_accueil.htm

Smith A. (2009), « À la recherche du territoire. Lecture critique de quatre ouvrages sur la France infranationale », Revue Française de Science Politique 58 (8), pp. 1019-1027.

Talbot D. (2008), «Les institutions créatrices de proximité », Revue d'économie régionale et urbaine, n 3, pp. 289-310. 
Résumé

\title{
La place des dynamiques territoriales dans la régulation de l'orientation scolaire
}

\author{
Par Thierry Berthet, Stéphanie Dechezelles, Rodolphe Gouin, Véronique Simon
}

La littérature scientifique française s'est peu interrogée sur les modes de régulation et la dimension territoriale de l'orientation scolaire. L'analyse des dynamiques territoriales de régulation et des pratiques d'orientation des élèves, proposée ici, articule trois perspectives - rôle historique des logiques territoriales dans la structuration du système français d'orientation, dynamiques des partenariats locaux et effets du caractère spatialisé des pratiques d'orientation - autour de l'hypothèse d'une forte autonomie locale des opérateurs de l'orientation scolaire.

\section{Mots clés}

Orientation scolaire-professionnelle, approche locale, partenaires sociaux Journal of Economic Literature: 121 